\title{
Pulmonary hemosiderosis in children with Down syndrome: a national experience
}

\author{
Aurelia Alimi ${ }^{1}$, Jessica Taytard', Rola Abou Taam², Véronique Houdouin ${ }^{3}$, Aude Forgeron ${ }^{4}$, Marc Lubrano Lavadera ${ }^{5}$, \\ Pierrick Cros $^{6}$, Isabelle Gibertini ${ }^{7}$, Jocelyne Derelle ${ }^{8}$, Antoine Deschildre $^{9}$, Caroline Thumerelle ${ }^{9}$, Ralph Epaud ${ }^{10}$, \\ Philippe Reix ${ }^{11}$, Michael Fayon ${ }^{12}$, Sylvie Roullaud ${ }^{13}$, Françoise Troussier ${ }^{14}$, Marie-Catherine Renoux ${ }^{15}$, \\ Jacques de Blic ${ }^{2}$, Sophie Leyronnas ${ }^{1}$, Guillaume Thouvenin ${ }^{1,16}$, Caroline Perisson ${ }^{1}$, Aimé Ravel ${ }^{17}$, \\ Annick Clement ${ }^{1,18}$, Harriet Corvol ${ }^{1,16}$, Nadia Nathan ${ }^{1,18^{*}}$ (D) and for the French RespiRare ${ }^{\circledast}$ group
}

\begin{abstract}
Background: Pulmonary hemosiderosis is a rare and complex disease in children. A previous study from the French RespiRare ${ }^{\circledast}$ network led to two important findings: $20 \%$ of the children presented with both pulmonary hemosiderosis and Down syndrome (DS), and at least one tested autoantibody was found positive in $50 \%$. This study investigates the relationships between pulmonary hemosiderosis and DS.

Methods: Patients younger than 20 years old and followed for pulmonary hemosiderosis were retrieved from the RespiRare ${ }^{\circledast}$ database. Clinical, biological, functional, and radiological findings were collected, and DS and non-DS patients' data were compared.

Results: A total of 34 patients (22 girls and 12 boys) were included, among whom nine (26\%) presented with DS. The mean age at diagnosis was $4.1 \pm 3.27$ years old for non-DS and $2.9 \pm 3.45$ years old for DS patients. DS patients tended to present a more severe form of the disease with an earlier onset, more dyspnoea at diagnosis, more frequent secondary pulmonary hypertension, and an increased risk of fatal evolution.

Conclusions: DS patients have a higher risk of developing pulmonary hemosiderosis, and the disease seems to be more severe in this population. This could be due to the combination of an abnormal lung capillary bed with fragile vessels, a higher susceptibility to autoimmune lesions, and a higher risk of evolution toward pulmonary hypertension. A better screening for pulmonary hemosiderosis and a better prevention of hypoxia in DS paediatric patients may prevent a severe evolution of the disease.
\end{abstract}

Keywords: Pulmonary hemosiderosis, Down syndrome, Children, Autoimmunity, Interstitial lung disease, Celiac disease, Vasculitis, Pulmonary hypertension

\section{Background}

Pulmonary hemosiderosis is a rare lung disease characterised by the triad hemoptysis, iron deficiency anaemia, alveolar and/or interstitial opacities on lung imaging. Bronchoalveolar lavage (BAL) and/or lung biopsy ascertain the diagnosis. The BAL fluid is bloody with a hemosiderin-laden macrophage ratio above $30 \%$ and/or

\footnotetext{
* Correspondence: nadia.nathan@aphp.fr

1 Assistance Publique Hôpitaux de Paris (APHP), Pediatric Pulmonology

department and Reference centre for rare lung diseases, RespiRare,

Trousseau Hospital, 75012 Paris, France

${ }^{18}$ Sorbonne Université, Inserm UMR-S933, Paris, France

Full list of author information is available at the end of the article
}

a Golde score higher than 50 [1]. Its frequency is poorly documented, but some authors hypothesised an incidence of 0.24-1.23 per million [2]. In children, very few cases are described worldwide. An association with celiac disease (Lane-Hamilton syndrome) and cow milk protein intolerance (Heiner syndrome) has been reported [3-6]. However, apart from the disease-specific condition, the aetiology and the pathophysiology of pulmonary hemosiderosis remain unknown. It is a chronic disease that commonly evolves in successive relapses separated by periods of remission. The prognosis is highly variable from a unique exacerbation with a complete recovery to multiple relapses with a risk of evolution toward lung fibrosis and

(c) The Author(s). 2018 Open Access This article is distributed under the terms of the Creative Commons Attribution 4.0 International License (http://creativecommons.org/licenses/by/4.0/), which permits unrestricted use, distribution, and 
terminal respiratory insufficiency. Corticosteroids are the mainstay of the treatment, with some children receiving also immunosuppressive drugs [7]. The French reference centre for rare lung diseases network RespiRare ${ }^{\bullet}$ previously reported a paediatric study population of 25 children aged 0.8 to 14 years old at diagnosis [8]. Two important findings were observed: 5 (20\%) children presented with both pulmonary hemosiderosis and Down syndrome (DS), a percentage higher than expected; and at least one tested autoantibody was found positive in 50\%. The present study aimed to investigate the newly documented relationships between pulmonary hemosiderosis and DS in the RespiRare ${ }^{\circ}$ network.

\section{Materials and methods Patients}

Prevalent patients with pulmonary hemosiderosis were retrieved from the national RespiRare ${ }^{\oplus}$ database with a query on the words pulmonary hemosiderosis, alveolar hemorrhage, siderophage, Golde score and hemoptysis. The database and data collection have been approved by French national data protection authorities (CNIL $\mathrm{n}^{\circ}$ 908,324 and CCTIRS n 08.015bis). Each patient and/or his or her legal representatives were informed prior to entering their data in the database. The charts of all the patients meeting the keywords were reviewed. Patients with a proven pulmonary hemosiderosis on BAL and/or lung biopsy between 1997 and 2017 were selected. Patients older than 20 years old at the time of the study were excluded.

\section{Data}

The following data were collected from the RespiRare database and analysed: age at diagnosis; gender; DS status; familial history of pulmonary hemosiderosis and/or autoimmune disease; initial symptoms; biological parameters, including hemoglobin $(\mathrm{Hb})$, reticulocytes, autoantibodies (antinuclear antibodies [ANA], anti-cytoplasmic antibodies [ANCA], anti-smooth muscle, anti-cyclic citrullinated peptide [CCP], anti-proteinase-3 [PR-3], anti-myeloperoxidase [MPO], anti-DNA, anti-endomysium, anti-transglutaminase, anti-SSA, anti-cardiolipin) and rheumatoid factor (RF); lung imaging results, including chest X-ray and thoracic high-resolution computed tomography (HRCT); pulmonary function tests (PFT); BAL and histological results; type and durations of treatments; and evolution of the disease with a specific attention to the presence or absence of pulmonary arterial hypertension (PAH) and relapses. Relapses were defined by the presence of a hemoptysis and/or a respiratory exacerbation (defined according to the ChiLD criteria) [9] associated with either new radiological findings compatible with an alveolar bleeding or increased anaemia or deglobulisation.

\section{Statistics}

The data from the pulmonary hemosiderosis patients with no DS, the non-DS group, were compared to those from the patients with both pulmonary hemosiderosis and DS, the DS group. Quantitative values were reported as median and range or mean and standard deviations. Qualitative data were reported as number (percentages). Comparisons between groups were established using a non-parametric $t$-test. $P$ values less than 0.05 were considered statistically significant.

\section{Results}

\section{Population clinical characteristics}

A total of 42 paediatric patients were followed for pulmonary hemosiderosis in the RespiRare network. Eight patients were excluded because they were older than 20 years. The main clinical characteristics of the 34 included patients are presented in Table 1 and Additional file 1: Table S1. The age at diagnosis ranged from 3 days to 11.5 years old (Fig. 1). Among the 34 included patients, 9 (26\%) presented with DS genetically confirmed (DS group); 8 had a free and homogeneous trisomy, and one patient had a partial trisomy with unbalanced translocation inherited from a balanced translocation in her mother.

In the non-DS group $(n=25)$, the sex ratio was in favour of girls $(72 \%)$. Three patients had a familial form of the disease (one had an uncle with pulmonary hemosiderosis and 2 were siblings), 2 had a biologically confirmed cow's milk allergy (one diagnosed at the same time as the pulmonary hemosiderosis and the other 5 years before), and one patient was also diagnosed with type B Niemann Pick disease. In this group, none of the patients presented with cardiac comorbidities.

In the DS group $(n=9)$, the sex ratio was in favour of boys $(56 \%)$. Two had a familial history of autoimmunity without pulmonary hemosiderosis, and 4 had a congenital cardiopathy. Two had identified PAH prior to the pulmonary hemosiderosis diagnosis. The other comorbidities are listed in Additional file 1: Table S1.

At diagnosis, dyspnoea was the most frequent symptom $(n=23,68 \%)$. Hemoptysis was documented in only $16(47 \%)$ of the patients (Table 1). The patients of the DS group presented with a higher frequency of dyspnoea $(100 \%$ in the DS group vs. $60 \%$ in the non-DS group, $P=0.04)$ and less hemoptysis $(22 \%$ vs. $56 \%$, respectively; $P=0.1$. Cough and pneumonia were also frequently reported at diagnosis in both groups.

\section{Investigations at diagnosis}

In both groups, most patients presented with a severe anaemia $(\mathrm{Hb}<7 \mathrm{~g} / \mathrm{dl})$. All the patients presented with an alveolar and interstitial pattern with a diffuse distribution of the lesions on the chest radiography and/or the HRCT scan (available for 28 [82\%] patients, Fig. 2). The main 
Table 1 Main characteristics of the patients with pulmonary hemosiderosis

\begin{tabular}{|c|c|c|c|c|}
\hline & All patients $n=34$ & Non-DS group $n=25$ & DS group $n=9$ & $P$-value \\
\hline & n (\%) & n (\%) & n (\%) & \\
\hline Girls & $22(65 \%)$ & $18(72 \%)$ & $4(44 \%)$ & 0.22 \\
\hline Boys & $12(35 \%)$ & $7(28 \%)$ & $5(56 \%)$ & 0.22 \\
\hline Mean age at the diagnosis & $3.80 \pm 3.30$ & $4.15 \pm 3.27$ & $2.92 \pm 3.45$ & 0.47 \\
\hline \multicolumn{5}{|l|}{ Family history } \\
\hline Pulmonary hemosiderosis & $3(9 \%)$ & $3(12 \%)$ & $0(0 \%)$ & 0.55 \\
\hline Autoimmune disorder & $2(6 \%)$ & $0(0 \%)$ & $2(22 \%)$ & 0.06 \\
\hline \multicolumn{5}{|l|}{ Personal history } \\
\hline $\mathrm{PAH}$ & $3(9 \%)$ & $0(0 \%)$ & $3(33 \%)$ & 0.01 \\
\hline Cardiopathy & $4(12 \%)$ & $0(0 \%)$ & $4(44 \%)$ & 0.003 \\
\hline \multicolumn{5}{|l|}{ Symptoms at presentation } \\
\hline Hemoptysis & $16(47 \%)$ & $14(56 \%)$ & $2(22 \%)$ & 0.13 \\
\hline Cough & $10(29 \%)$ & $8(32 \%)$ & $2(22 \%)$ & 0.69 \\
\hline Dyspnoea & $23(68 \%)$ & $15(60 \%)$ & $9(100 \%)$ & 0.03 \\
\hline Pneumonia & $9(26 \%)$ & $6(24 \%)$ & $3(33 \%)$ & 0.67 \\
\hline \multicolumn{5}{|l|}{ Minimal hemoglobin } \\
\hline$<7 \mathrm{~g} / \mathrm{dl}$ & $20(62.5 \%)$ & $14^{*}(61 \%)$ & $6(56 \%)$ & 1 \\
\hline$\geq 7 \mathrm{~g} / \mathrm{dl}$ & $12(37.5 \%)$ & $9^{*}(39 \%)$ & $3(44 \%)$ & 1 \\
\hline
\end{tabular}

Abbreviations: DS down syndrome, $P A H$ pulmonary arterial hypertension

${ }^{*}$ missing data for 2 patients

abnormalities were ground-glass opacities, nodules, and alveolar condensations. Lung fibrosis was already present at the first evaluation for 2 patients (one in each group).

All BAL were consistent with the diagnosis of pulmonary hemosiderosis, with a median of $83 \%$ of hemosiderin-laden macrophages and/or a median Golde score of 168. Four patients (all in the non-DS group) underwent an open lung biopsy with a positive Perls' staining for all.

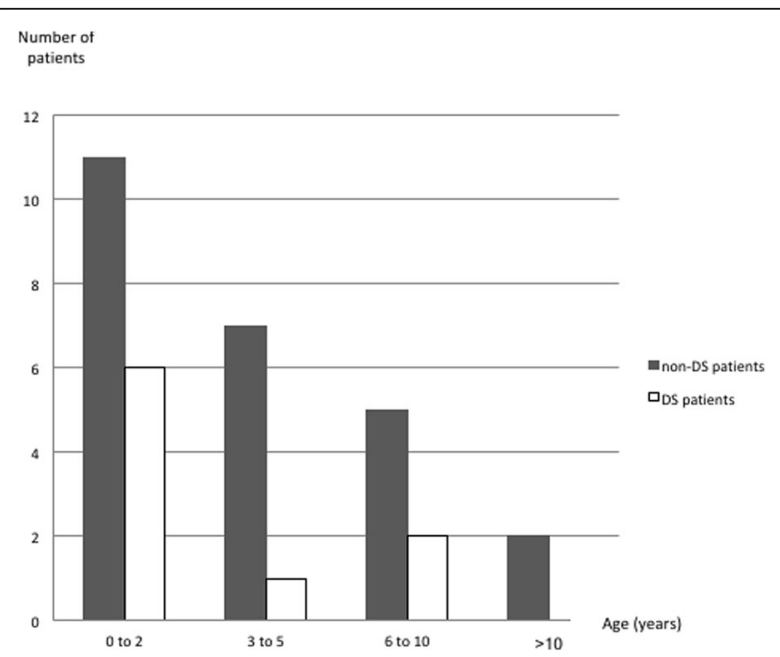

Fig. 1 Age at presentation of the 34 included patients. Black bars represent the ages at presentation of the non-DS patients, and white bars represent the ages at presentation of the DS patients
At diagnosis, only 13 of the 34 patients were able to perform PFT because of their young age or their general or respiratory condition. PFT were normal in 7 (54\%) patients and showed a restrictive, an obstructive, or a mixed syndrome in, respectively, 2 (15\%), 1 (8\%), and 2 (15\%) patients. Diffusion capacity of the lung for carbon monoxide (DLCO) was measured in 4 patients and was below expected values $(<75 \%)$ for 3 of them, 2 and 1 in the non-DS group and the DS group, respectively.

As previously described, a large number of the patients presented with biological signs of autoimmunity (Table 2): 24 (75\%) patients had at least one positive antibody, 18 (78\%) in the non-DS group and 6 (67\%) in the DS group. In both groups, ANA were the most frequently observed antibodies $(n=11,32 \%)$; the other positive antibodies were ANCA, anti-smooth muscle, RF, anti-CCP, antiPR-3, anti-MPO, anti-DNA, anti-endomysium, antitransglutaminase, anti-SSA and anti-cardiolipin. Their repartition between both groups is listed in Fig. 3 .

\section{Treatment}

Treatment information was available for 32 patients. All the patients received systemic corticosteroids as first-line treatment. Monthly intravenous pulses of methylprednisolone $\left(300 \mathrm{mg} / \mathrm{m}^{2} /\right.$ day for 3 days) were used for four (12\%) patients, oral corticosteroids (1 to $2 \mathrm{mg} / \mathrm{kg} /$ day) for $9(28 \%)$ patients, and both for 19 (59\%) patients. A long-term treatment was necessary for the majority of 


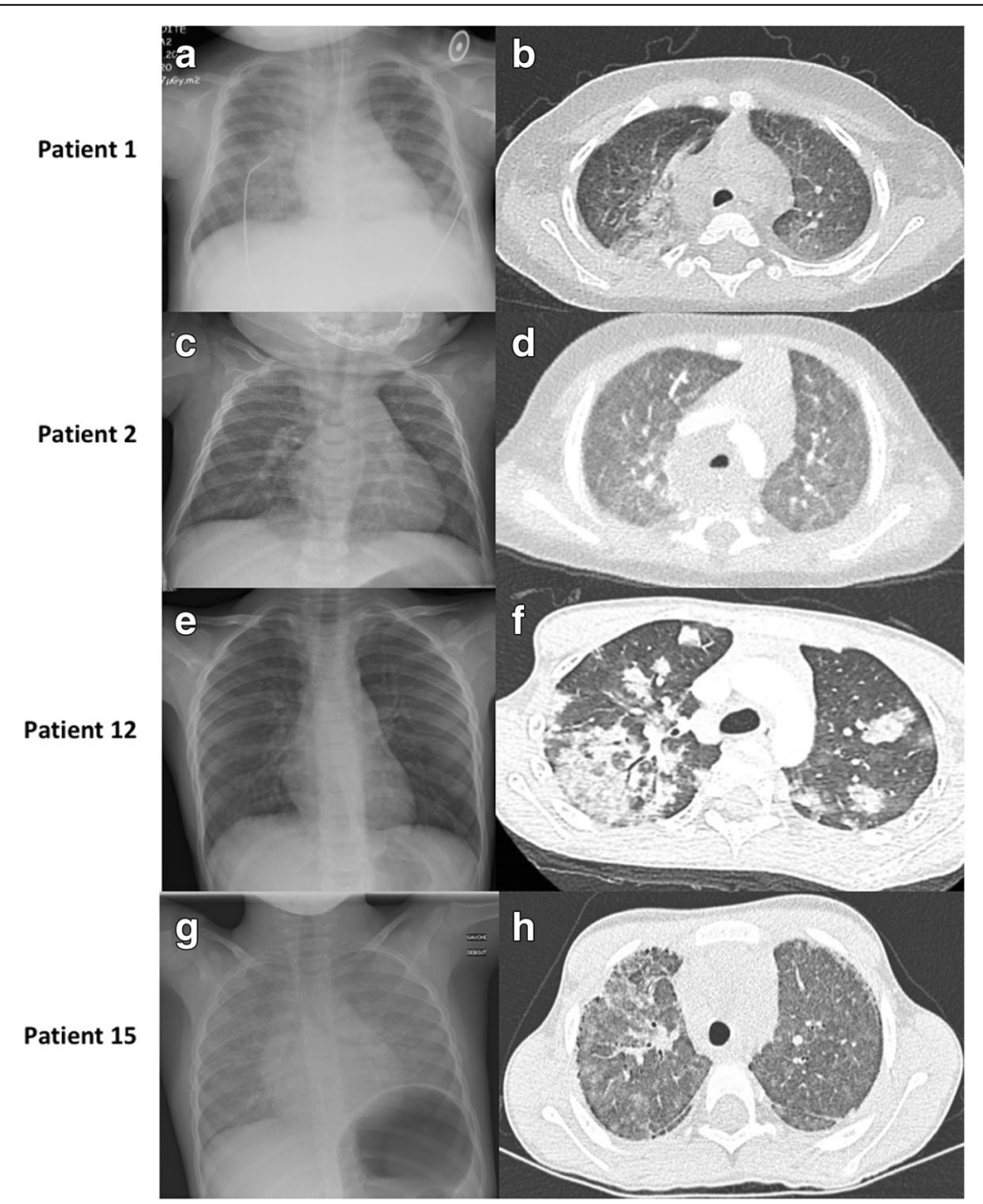

Fig. 2 Lung imaging of two patients in the DS group (patients 1 and 2), and two in the non-DS group (patients 12 and 15). Panels a and $\mathbf{b}$ Chest $x$-ray and thoracic HRCT-scan of patient 1 at diagnosis (8 months of age) show bilateral alveolar opacities with a posterior predominance and diffuse ground glass opacities. Panels $\mathbf{c}$ and $\mathbf{d}$ Chest $\mathrm{x}$-ray and thoracic HRCT-scan of patient two at 1 month of age show bilateral diffuse ground glass opacities. Panels $\mathbf{e}$ and $\mathbf{f}$ Chest $\mathrm{x}$-ray and thoracic HRCT-scan of patient 12 at 4.3 years old show bilateral alveolar condensations with a patchy repartition, central and peripheral, and surrounding ground glass opacifications. Panels $\mathbf{g}$ and $\mathbf{h}$ Chest $\mathrm{x}$-ray and thoracic HRCT-scan of patient 15 at 5 years old show bilateral patchy ground glass opacifications and signs of lung fibrosis with reticulations and sub-pleural cysts

them, with $30 \%$ of the patients still receiving corticosteroids after 1 year of follow-up, and $25 \%$ after 5 years. Because the three deceased patients belonged to the DS group, the duration of the corticosteroid treatment could not be compared between both groups.
More than 25\% of the patients were treated with second-line therapies. A total of ten patients received hydroxychloroquine (31\%): nine patients in the non-DS group and one in the DS group. Immunosuppressive drugs (mycophenolate mofetil, cyclophosphamide and/or

Table 2 Positive antibodies at diagnosis in the pulmonary hemosiderosis cohort

\begin{tabular}{lllll}
\hline Positive antibodies & Total (32 patients) & non-DS group (23 patients) & \multicolumn{2}{l}{$\begin{array}{l}\text { DS group (9 patients) } \\
\mathrm{n}(\%)\end{array}$} \\
\hline 1 & $\mathrm{n}(\%)$ & $9(39 \%)$ & $3(33 \%)$ & 0.78 \\
2 & $12(34 \%)$ & $6(26 \%)$ & $1(11 \%)$ & 0.64 \\
$>2$ & $7(19 \%)$ & $3(13 \%)$ & $2(22 \%)$ & 0.60 \\
Total & $5(12 \%)$ & $18(78 \%)$ & $6(67 \%)$ & 0.04 \\
\hline
\end{tabular}

Abbreviations: DS down syndrome

*Missing data for 2 patients in the non-DS group 


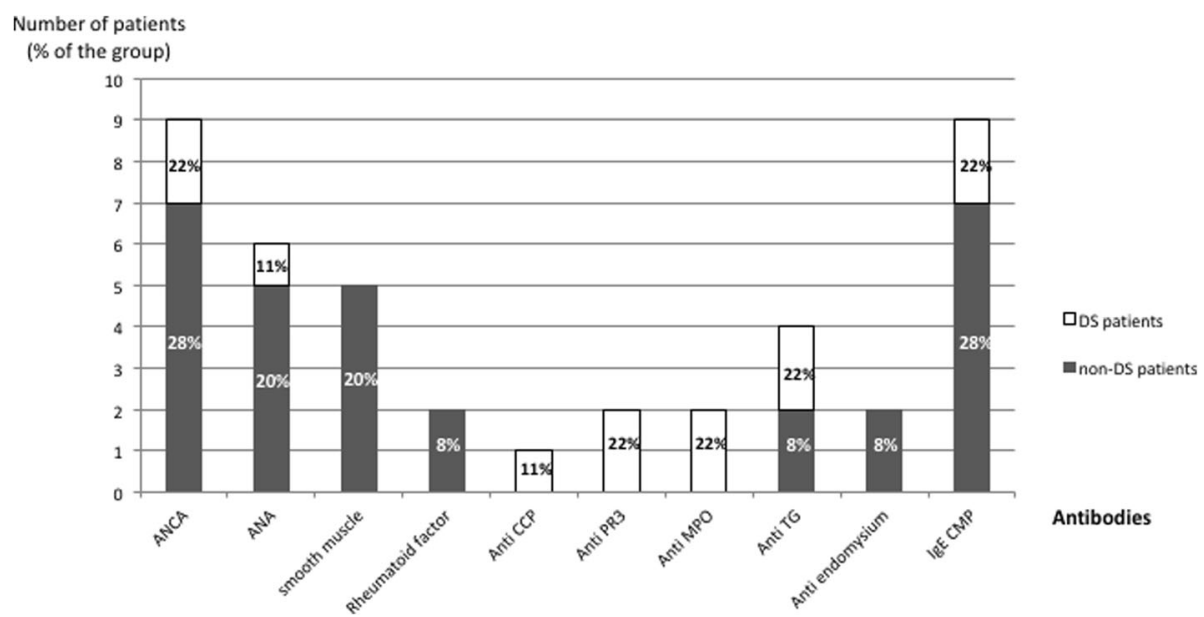

Fig. 3 Number of patients with positive antibodies in each group. The black bars represent the number of non-DS patients with positive antibodies and the white bars represent the number of DS patients with positive antibodies. The $\%$ in each bar is the \% of patients in each group with positive antibodies. Abbreviations: DS = Down syndrome, ANCA = anti-cytoplasmic antibodies; ANA = antinuclear antibodies; CCP = anti-cyclic citrullinated peptide; PR3 = anti-proteinase 3; MPO = anti-myeloperoxidase; TG = anti-transglutaminase; DS = Down syndrome

azathioprine) were used for nine patients (28\%): six (26\%) in the non-DS group and three (33\%) in the DS group. Beside drugs, patients with cow's milk protein allergies were treated with an exclusion diet [10].

\section{Evolution}

The mean length of follow-up was 4.9 [0.8-18.3] years; most of the patients $(n=25,73.5 \%)$ were followed for more than 3 years. For 13 (40.6\%) patients, pulmonary hemosiderosis occurred only as a single event, with no relapse after treatment (Table 3). Nineteen patients (59.3\%) experienced at least one relapse: 13 (56.5\%) patients in the non-DS group and six (67\%) in the DS group $(P=0.63)$. Five patients, all in the DS group, presented with a PAH. The PAH pre-existed the pulmonary hemosiderosis for two and was secondary for three patients. Three of them died after multiple relapses from acute $\mathrm{PAH}$ and/or massive pulmonary hemorrhage at age 0.7, 2 and 7 years old, respectively. For these three patients, the pulmonary hemosiderosis was diagnosed within the first months of life.

\section{Discussion}

Pulmonary hemosiderosis is a very rare disease in children, and its pathophysiology remains unclear. We report here our national experience through the RespiRare network. This study highlights the surprising over-representation of DS in pulmonary hemosiderosis paediatric patients. DS is the most common genetic disorder, with a prevalence reaching 140 per 100,000 children [11]. Therefore, in our pulmonary hemosiderosis cohort population of 34 patients, 0 to 1 patient with DS was expected. However, nine children, i.e., around a quarter of the pulmonary hemosiderosis population, presented with DS. Pulmonary hemosiderosis in DS patients has not been reported on so far, except through isolated case reports $[12,13]$. Moreover, based on our national findings, the estimated prevalence of pulmonary hemosiderosis in children reaches 1.85 per $1,000,000$ children, compared to 138.5 per 1,000,000 DS children.

Patients with and without DS displayed remarkable differences. In the DS group, six patients out of 9 were younger than 3 years old at diagnosis, whereas in the non-DS patients, two-thirds of the patients were older than 3 years old $[8,14-16]$. Although hemoptysis is a

Table 3 Evolution of the patients with pulmonary hemosiderosis

\begin{tabular}{lllll}
\hline & $\begin{array}{l}\text { Total (32 patients) } \\
\mathrm{n}(\%)\end{array}$ & $\begin{array}{l}\text { non-DS group (23 patients) } \\
\mathrm{n}(\%)\end{array}$ & $\begin{array}{l}\text { DS group (9 patients) } \\
\mathrm{n}(\%)\end{array}$ \\
\hline No relapses & $13(40.6 \%)$ & $10(43.4 \%)$ & $3(33.3 \%)$ & 0.7 \\
Relapses & $19(59.3 \%)$ & $13(56.5 \%)$ & $6(67 \%)$ & 0.46 \\
Pulmonary arterial hypertension & $5(14.7 \%)$ & 0 & $5(55 \%)$ & 0.0003 \\
Death & $3(9 \%)$ & 0 & $3(33 \%)$ & 0.0003 \\
\hline
\end{tabular}

Abbreviations: DS down syndrome

*Missing data for 2 patients in the non-DS group 
classic sign of the disease, it was present in fewer than half of the patients, whereas dyspnoea was the most frequent respiratory symptom. DS patients seemed to present a more severe form of the disease with an earlier onset, more dyspnoea at diagnosis, more secondary PAH and a major risk of fatal evolution.

In this study, autoimmunity stigma was documented in a large majority of the patients in both groups $(75 \%$, $n=24)$. The link between pulmonary hemosiderosis and the presence of circulating autoantibodies is not clearly understood in patients with no valid diagnosis criteria for vasculitis [8]. DS, particularly in men, is known to be associated with a high incidence of autoimmune disorders such as thyroiditis, hypothyroidism, type 1 diabetes, Addison disease, celiac disease, and other, rarer, disorders, including primary sclerosing cholangitis [17]. Lungs are not considered a privileged target for DS autoimmunity, but autoantibodies are frequently found in DS patients even with no evidence of clinical autoimmune disease [18]. Recent studies reported the crucial role in DS autoimmune dysfunction of the autoimmune regulator protein (AIRE) located on chromosome 21 . AIRE is selectively expressed in the thymus and is a transcription factor for many tissue-restricted antigens that enhance the generation of regulatory T-cells and consecutively induce a central tolerance. It is presumed to protect against autoimmune diseases. Bi-allelic mutations of AIRE are associated with an autoimmune disease that is similar to the spectrum of autoimmunity observed in DS [19]. In DS, despite three expressed copies of AIRE, the overall AIRE expression was shown to be reduced compared to controls. All together, these findings favour a central role of AIRE in DS autoimmune disorders $[20,21]$. Autoimmunity could be one of the links between DS and pulmonary hemosiderosis. In our study, several antibodies were found exclusively in DS or non-DS patients, but the majority of the patients had positive circulating antibodies, with no significant differences between groups. Surprisingly, DS patients did not receive more immunosuppressive agents than those of the non-DS group $(P=0.41)$. The reasons for fewer prescriptions of immunosuppressive drugs in DS patients are unclear. A hypothesis could be that clinicians were avoiding the risk of major sensitivity to chemotherapy in DS patients [22-24].

Another hypothesis to explain the association between pulmonary hemosiderosis and DS could be an altered alveolar and vascular development of the lungs. It is known that children with DS have more microscopic pulmonary malformations and present an increased risk for PAH development, independently from cardiac malformations [25]. Histological descriptions have shown elements in favour of arrested lung development such as alveolar simplification, persistence of a double capillary network, prominence of a bronchial circulation or, more recently, intrapulmonary bronchopulmonary anastomoses [26]. Lung epithelial development is closely related to signalling from the vascular compartment: an inhibition of the vascular endothelial growth factor (VEGF) induces an altered angiogenesis and an abnormal alveolar structure development in the foetus [27]. Several anti-angiogenic factors are located on chromosome 21: endostatin (COL18A1), beta-amyloid protein $(A P P)$, and regulator of calcineurin 1 (RCAN1). These factors are overexpressed during the DS foetal period due to the three copies of the genes. It has recently been shown that their up-regulation in DS lung tissues was associated with a reduced vessel density and an increase of the vessel wall thickness compared to non-DS lung tissues [28]. The in-utero capillary development of the DS foetus is consistent with the hypothesis of an altered maturation of the capillary network of the alveoli and the absence of regression of the thick arterial musculature of the pulmonary vessels [29]. This impaired vascular development could be responsible for an altered alveolar maturation with simplified large alveoli. The reduced total alveolar surface associated with an abnormal capillary network could constitute a risk factor for hypoxemia, $\mathrm{PAH}$ and alveolar hemorrhage.

Patients with DS have additional risk factors for PAH due to chronic hypoxia and recurrent hypoxic events such as frequent congenital heart diseases, lung infections, recurrent aspirations, and obstructive sleep apnea syndrome (OSAS) [30]. OSAS is observed in up to half of adult DS patients [31]. In children, extreme prevalence between 0 and $100 \%$ has been reported in small cohorts [30, 32, 33]. Multiple factors can explain OSAS in children with DS, but the main causes include hypotonia, facial dysmorphia with macroglossia and narrow upper airways. It has also been suggested that tonsillar growth in the first months of life could increase the airway collapse [34]. Central apnoea reported in DS patients can also increase the OSAS severity. Untreated OSAS increases chronic hypoxia and, subsequently, PAH development. Altogether, in DS patients, the severity of pulmonary hemosiderosis could be due to the combination of a higher susceptibility to autoimmune lesions of the alveolar capillary, an abnormal lung capillary bed and a higher PAH risk. These pathophysiologic hypotheses could shed further new light on possible abnormal lung maturation in non-DS patients with pulmonary hemosiderosis.

\section{Conclusion}

This study reports for the first time a higher risk of severe pulmonary hemosiderosis in DS paediatric patients. Because alveolar bleeding symptoms can be inconspicuous, it could be suggested to perform a chest X-ray in all DS patients with chronic unexplained anaemia and/or chronic, unexplained dyspnoea. At this stage, only hypotheses can be proposed on the links between DS and 
pulmonary hemosiderosis such as an increased risk of $\mathrm{PAH}$. If such a hypothesis is confirmed by further studies, systematic sleep investigations in DS patients could be proposed to screen for OSAS and to prevent PAH. For all pulmonary hemosiderosis patients, with or without DS, autoimmune explorations are critical at diagnosis and may be repeated regularly. In the era of genomic research, DS patients' aggregation in such a rare disease could be a real opportunity to link chromosome 21 genes to new pathophysiologic clues for pulmonary hemosiderosis.

\section{Additional file}

Additional file 1: Table S1. Detailed characteristics of the 34 included patients. (DOCX $24 \mathrm{~kb}$ )

\section{Abbreviations}

AIRE: Autoimmune regulator protein; ANA: Antinuclear antibodies; ANCA: Anti-cytoplasmic antibodies; APP: Beta-amyloid protein; BAL: Bronchoalveolar lavage; CCP: Cyclic citrullinated peptide; COL18A1: Gene encoding the endostatin; CS: Corticosteroids; DLCO: Diffusion capacity of the lung for carbon monoxide; DS: Down syndrome; F: Female; Hb: Hemoglobin; HCQ: Hydroxychloroquine; HRCT: High-resolution computed tomography; M: Male; MD: Missing data; MMF: Mycophenolate mofetil; MPO: Myeloperoxidase; OSAS: Obstructive sleep apnea syndrome; PAH: Pulmonary arterial hypertension; PFT: Pulmonary function tests; PR3: Proteinase 3; RCAN1: Regulator of calcineurin 1; RF: Rheumatoid factor; TG: Transglutaminase; VEGF: Vascular endothelial growth factor

\section{Acknowledgments}

We thank the Assistance Publique Hôpitaux de Paris, Sorbonne Université Paris, France, and the national networks for rare lung diseases: Centre de référence des maladies respiratoires rares (RespiRare), Centre de référence des maladies pulmonaires rares (OphaLung) and Filière de soins pour les maladies respiratoires rares (RespiFIL). We also thank the Jerôme Lejeune Institute for Down syndrome. The ILD cohort is developed in collaboration with the Rare Cohort Disease (RaDiCo)-ILD project (ANR-10-COHO-0003), the FP7-305653-child-EU project and the COST Action European network for translational research in children's and adult interstitial lung disease (COST-ILD) project (CA16125).

\section{Availability of data and materials}

The data supporting the results reported in this article are extracted from the national database RespiRare and can be available under request.

\section{Authors' contributions}

AA and NN collected the data and wrote the manuscript. NN, JT, HC, GT, CP, $S L, A C$ reviewed the manuscript. RAT, VH, AF, ML, PC, IG, JD, AD, CT, RE, PR, $M F, F T, M C R, J d B, A R$ provided patients data, and reviewed the manuscript. AR provided his expertise on Down syndrome. All authors read and approved the final manuscript.

\section{Ethics approval and consent to participate}

The database and data collection have been approved by French national data protection authorities (CNIL n908,324 and CCTIRS n08.015bis). Each patient and/or his or her legal representatives were informed prior to entering their data in the database.

\section{Competing interests}

The authors declare that they have no competing interests.

\section{Publisher's Note}

Springer Nature remains neutral with regard to jurisdictional claims in published maps and institutional affiliations.

\section{Author details}

Assistance Publique Hôpitaux de Paris (APHP), Pediatric Pulmonology department and Reference centre for rare lung diseases, RespiRare, Trousseau Hospital, 75012 Paris, France. ${ }^{2}$ APHP, Pediatric Pulmonology department, RespiRare, Necker Enfants Malades Hospital , 75015 Paris, France. ${ }^{3}$ APHP, Pediatric Pulmonology department, RespiRare, Faculty Paris Diderot VII, Inserm U1 149, Robert Debré Hospital, Paris, France. ${ }^{4}$ Pediatric department, Hospital Center, Inserm U646, 72037 Le Mans, France. ${ }^{5}$ Pediatric Pulmonology department, University Hospital, Rouen, France. ${ }^{6}$ Pediatric Pulmonology department, University Hospital, Inserm 1078, Brest, France. ${ }^{7}$ Pediatric department, University Hospital, Tours, France. ${ }^{8}$ Pediatric department, University Hospital, Nancy, France. ${ }^{9}$ Pediatric Pulmonology department, University Hospital, UMR CNRS 8204 - Inserm U1019, Lille, France. ${ }^{10}$ Pediatric Pulmonology department, RespiRare, Créteil University Hospital, Inserm U955, Créteil, France. ${ }^{11}$ Pediatric Pulmonology department, University Hospital, UMR CNRS 5558, Lyon, France. ${ }^{12}$ Pediatric Pulmonology department, University Hospital, U1219, Bordeaux, France. ${ }^{13}$ Pediatric department, Hospital Centre, Angouleme, France. ${ }^{14}$ Pediatric Pulmonology department, University Hospital, Inserm U892, Angers, France. ${ }^{15}$ Pediatric Pulmonology department, University Hospital, Inserm U1046, Montpellier, France. ${ }^{16}$ Sorbonne Université, Inserm UMR-S938, Paris, France. ${ }^{17}$ Institut Jérôme Lejeune, Paris, France. ${ }^{18}$ Sorbonne Université, Inserm UMR-S933, Paris, France.

Received: 2 October 2017 Accepted: 12 April 2018

Published online: 20 April 2018

\section{References}

1. Drew WL, Finley TN, Golde DW. Diagnostic lavage and occult pulmonary hemorrhage in thrombocytopenic immunocompromised patients. Am Rev Respir Dis. 1977;116(2):215-21.

2. Bakalli I, Kota L, Sala D, et al. Idiopathic pulmonary hemosiderosis - a diagnostic challenge. Ital J Pediatr. 2014;40:35.

3. Khemiri M, Ouederni M, Khaldi F, Barsaoui S. Screening for celiac disease in idiopathic pulmonary hemosiderosis. Gastroenterol Clin Biol. 2008;32(8-9):745-8.

4. Lane DJ, Hamilton WS. Idiopathic steatorrhoea and idiopathic pulmonary haemosiderosis. Br Med J. 1971:2(5753):89-90.

5. Moissidis I, Chaidaroon D, Vichyanond P, Bahna SL. Milk-induced pulmonary disease in infants (Heiner syndrome). Pediatr Allergy Immunol. 2005;16(6):545-52.

6. Heiner DC. Respiratory diseases and food allergy. Ann Allergy. 1984;53(6 Pt 2):657-64.

7. Chin CIC, Kohn SL, Keens TG, Margetis MF, Kato RM. A physician survey reveals differences in management of idiopathic pulmonary hemosiderosis. Orphanet J Rare Dis. 2015;10:98.

8. Taytard J, Nathan N, de BJ, et al. New insights into pediatric idiopathic pulmonary hemosiderosis: the French RespiRare $\left(^{(}\right)$cohort. Orphanet J Rare Dis. 2013;8:161.

9. Clement A, de BJ, Epaud R, et al. Management of children with interstitial lung diseases: the difficult issue of acute exacerbations. Eur Respir J. 2016:48(6):1559-63

10. Sethi GR, Singhal KK, Puri AS, Mantan M. Benefit of gluten-free diet in idiopathic pulmonary hemosiderosis in association with celiac disease. Pediatr Pulmonol. 2011;46(3):302-5.

11. Mégarbané A, Ravel A, Mircher $C$, et al. The 50th anniversary of the discovery of trisomy 21: the past, present, and future of research and treatment of Down syndrome. Genet Med. 2009;1 1(9):611-6.

12. Tenenbaum A, Malkiel S, Wexler ID, Levy-Khademi F, Revel-Vilk S, Stepensky P. Anemia in children with Down syndrome. Int J Pediatr. 2011;2011:813541.

13. Watanabe $H$, Ayusawa M, Kato M, et al. Idiopathic pulmonary hemosiderosis complicated by Down syndrome. Pediatr Int Off I Jpn Pediatr Soc. 2015;57(5):1009-12

14. Mourad AA, Parekh H, Bahna SL. A 17-month-old patient with severe anemia and respiratory distress. Allergy Asthma Proc. 2015;36(6):506-11.

15. Kabra SK, Bhargava S, Lodha R, Satyavani A, Walia M. Idiopathic pulmonary hemosiderosis: clinical profile and follow up of 26 children. Indian Pediatr. 2007:44(5):333-8.

16. Nuesslein $\mathrm{TG}$, Teig $\mathrm{N}$, Rieger $\mathrm{CH}$. Pulmonary haemosiderosis in infants and children. Paediatr Respir Rev. 2006;7(1):45-8.

17. Bull MJ. Committee on genetics. Health supervision for children with Down syndrome. Pediatrics. 2011;128(2):393-406. 
18. Nisihara RM, Skare TL, Silva MBG, et al. High positivity of anti-CCP antibodies in patients with Down syndrome. Clin Rheumatol. 2007;26(12):2031-5.

19. Mathis D, Benoist C. Aire. Annu Rev Immunol. 2009;27:287-312.

20. Skogberg $G$, Lundberg V, Lindgren $S$, et al. Altered expression of autoimmune regulator in infant Down syndrome thymus, a possible contributor to an autoimmune phenotype. J Immunol Baltim Md 1950. 2014;193(5):2187-95.

21. Giménez-Barcons $M$, Casteràs $A$, Armengol $M$ del $P$, et al. Autoimmune predisposition in Down syndrome may result from a partial central tolerance failure due to insufficient intrathymic expression of AIRE and peripheral antigens. J Immunol Baltim Md 1950. 2014;193(8):3872-9.

22. Meyr F, Escherich G, Mann G, et al. Outcomes of treatment for relapsed acute lymphoblastic leukaemia in children with Down syndrome. $\mathrm{Br} J$ Haematol. 2013;162(1):98-106.

23. Peeters M, Poon A. Down syndrome and leukemia: unusual clinical aspects and unexpected methotrexate sensitivity. Eur J Pediatr. 1987;146(4):416-22.

24. Taub JW, Ge Y. Down syndrome, drug metabolism and chromosome 21. Pediatr Blood Cancer. 2005;44(1):33-9.

25. Saji T. Clinical characteristics of pulmonary arterial hypertension associated with Down syndrome. Pediatr Int Off J Jpn Pediatr Soc. 2014;56(3):297-303.

26. Bush D, Abman SH, Galambos C. Prominent intrapulmonary bronchopulmonary anastomoses and abnormal lung development in infants and children with Down syndrome. J Pediatr. 2017;180:156-162.e1.

27. Le Cras TD, Markham NE, Tuder RM, Voelkel NF, Abman SH. Treatment of newborn rats with a VEGF receptor inhibitor causes pulmonary hypertension and abnormal lung structure. Am J Physiol Lung Cell Mol Physiol. 2002;283(3):L555-62.

28. Reynolds $L E$, Watson $A R$, Baker $M$, et al. Tumour angiogenesis is reduced in the Tc1 mouse model of Down's syndrome. Nature. 2010;465(7299):813-7.

29. Galambos C, Minic AD, Bush D, et al. Increased lung expression of antiAngiogenic factors in Down syndrome: potential role in abnormal lung vascular growth and the risk for pulmonary hypertension. PLoS One. 2016;11(8):e0159005.

30. Konstantinopoulou S, Tapia IE, Kim JY, et al. Relationship between obstructive sleep apnea cardiac complications and sleepiness in children with Down syndrome. Sleep Med. 2016;17:18-24.

31. Hill EA. Obstructive sleep apnoea/hypopnoea syndrome in adults with Down syndrome. Breathe Sheff Engl. 2016;12(4):e91-6.

32. Levanon A, Tarasiuk A, Tal A. Sleep characteristics in children with Down syndrome. J Pediatr. 1999;134(6):755-60.

33. Dyken ME, Lin-Dyken DC, Poulton S, Zimmerman MB, Sedars E. Prospective polysomnographic analysis of obstructive sleep apnea in Down syndrome. Arch Pediatr Adolesc Med. 2003;157(7):655-60.

34. Hill CM, Evans $\mathrm{HJ}$, Elphick $\mathrm{H}$, et al. Prevalence and predictors of obstructive sleep apnoea in young children with Down syndrome. Sleep Med. 2016;27-28:99-106.

\section{Ready to submit your research? Choose BMC and benefit from:}

- fast, convenient online submission

- thorough peer review by experienced researchers in your field

- rapid publication on acceptance

- support for research data, including large and complex data types

- gold Open Access which fosters wider collaboration and increased citations - maximum visibility for your research: over $100 \mathrm{M}$ website views per year

At BMC, research is always in progress.

Learn more biomedcentral.com/submissions 